\title{
CLINICAL PRACTICE Exercise in Clinical Reasoning Pivot and Cluster: An Exercise in Clinical Reasoning
}

\author{
Jori E. May, $M D^{7}$, Reaford J. Blackburn, $M D^{2}$, Robert M. Centor, MACP, $M D^{1,3}$, and \\ Gurpreet Dhaliwal, $M D^{4,5}$
}

'Department of Medicine , University of Alabama at Birmingham, Birmingham, AL, USA; ${ }^{2}$ Lake Cumberland Regional Hospital, Somerset, KY, USA; ${ }^{3}$ Medical Services, Birmingham VA Medical Center, Birmingham, AL, USA; ${ }^{4}$ Department of Medicine, University of California San Francisco, San Francisco, CA, USA; ${ }^{5}$ Medical Service, San Francisco VA Medical Center, San Francisco, CA, USA.

KEY WORDS: clinical reasoning; urticaria; whiteboard; pivot and cluster. J Gen Intern Med 33(2):226-30

DOI: $10.1007 / \mathrm{s} 11606-017-4216-6$

(C) Society of General Internal Medicine 2017

I n this series, a clinician extemporaneously discusses the diagnostic approach (regular text) to sequentially presented clinical information (bold). Additional commentary on the diagnostic reasoning process (italics) is integrated throughout the discussion.

A 68-year-old woman presented to primary care with 1 year of intermittent fever up to $39{ }^{\circ} \mathrm{C}$, nausea, and an unintentional 80-pound weight loss. She also had drenching night sweats multiple times per week.

Sustained fever is generally caused by infectious, malignant, or autoimmune disorders. Initial considerations in this case would include human immunodeficiency virus (HIV), tuberculosis, lymphoma, and hyperthyroidism. Occasionally, autoinflammatory disorders may be responsible. In contrast to autoimmune disorders, which arise from antigen-dependent activation of the adaptive immune system, autoinflammatory disorders are characterized by antigen-independent activation of the innate immune system. Medications can cause fever as well, but a duration of months is unlikely.

Any case that is a challenge to diagnose is also a challenge to teach. At the whiteboard, a teacher can illustrate important principles of diagnostic reasoning. At this early stage, the teacher may list broad categories of disease, as the discussant did, and challenge learners to identify an exemplar for each category.

In the year prior to presentation, she was evaluated by multiple subspecialists. Extensive testing for infection, malignancy, and autoimmunity only revealed subcentimeter lymphadenopathy throughout the chest, abdomen, and pelvis on computerized tomography (CT) scan and an antinuclear antibody (ANA) titer of 1:160. During this time, she also developed progressive gait instability with frequent falls.

Received April 4, 2017

Revised August 4, 2017

Accepted October 30, 2017

Published online November 28, 2017
Mild lymphadenopathy and a moderately elevated ANA are both markers of an activated immune system that can arise from infections, malignancies, and autoimmune disorders.

Gait instability and falls are frequently multifactorial and may arise from a combination of motor and sensory deficits, changes in cognition and attentiveness, deconditioning and musculoskeletal degeneration, non-neurologic comorbidities such as cardiac and pulmonary disorders, and medication effects. Examples of gait instability that overlap with weight loss and fevers include paraneoplastic neuropathy or cerebellar dysfunction, a slowly compressive myelopathy from spinal tuberculosis, or a vasculitic neuropathy.

As the teacher continues to list notable but nonspecific findings on the whiteboard such as mild lymphadenopathy, ANA seropositivity, and gait instability, she can note how these findings do not effectively prioritize the categories of disease under consideration. The teacher may note the need for a diagnostic foothold, a problem around which a more focused differential diagnosis can be built.

The patient had type 2 diabetes mellitus (hemoglobin A1c 7.0\%), hypertension, and chronic urticaria. Five years before this presentation, she developed right hip and leg pain along with more diffuse, poorly localized pains. Magnetic resonance imaging (MRI) revealed a sclerotic lesion in the right femur. A femur biopsy was negative for malignancy. She was diagnosed with fibromyalgia. Her medications included cetirizine, metformin, losartan, hydrochlorothiazide, and ondansetron. She denied tobacco, alcohol, or illicit drug use.

Diabetes, hypertension, chronic urticaria, and bone lesions are all common conditions, but the latter two have conceivable overlap with systemic inflammation. Even after a diligent search for offending allergens such as insects, drugs, or environmental exposures, most chronic urticaria is labeled idiopathic; a few systemic diseases including urticarial vasculitis, mastocytosis, and Schnitzler's syndrome (a rare autoinflammatory syndrome) feature urticaria. Sclerotic bone lesions are caused by metastases and metabolic bone diseases (e.g., Paget disease of bone), but the majority are benign bone islands.

Within the context of nonspecific inflammation and its expansive differential diagnosis, the case now presents a diagnostic clue with a limited differential diagnosis: chronic urticaria. This serves as a diagnostic foothold. As the teacher 
builds a differential diagnosis for chronic urticaria, a common strategy would be to compose a vertical list on the whiteboard. An alternative approach utilizes a more comprehensive visual representation of a developing differential diagnosis: the pivot and cluster strategy (PCS). Using PCS, a clinician identifies an initial diagnosis which serves as the "pivot" around which she develops a collection of alternative diagnoses to that anchor point, which forms the "cluster." 1

The teacher designates the most common attribution for chronic urticaria, idiopathic urticaria, as the pivot on the whiteboard. Around this pivot, the teacher constructs a cluster of other causes of chronic urticaria including urticarial vasculitis, mastocytosis, and Schnitzler's syndrome (Fig. 1). A teacher could alternatively choose to illustrate a cluster for sclerotic bone lesions, which also presents a restricted differential diagnosis, but a single diagnosis is presented for simplicity.

The patient's temperature was $36.9^{\circ} \mathrm{C}$, blood pressure $117 / 73 \mathrm{mmHg}$, heart rate 82 beats per minute, respiratory rate 14 breaths per minute, and oxygen saturation $99 \%$ while breathing ambient air. She appeared chronically ill with bilateral temporal wasting. She was fully oriented. Her cardiopulmonary examination was normal. Her abdomen was soft and flat. The liver edge extended two centimeters below the costal margin. There was a non-tender, mobile $2-\mathrm{cm}$ lymph node in her left axilla. All joints were normal without erythema, effusion, or limitation of movement.

The neurologic examination disclosed the following abnormalities: decreased vibratory and proprioceptive sensation in her feet, symmetrically decreased reflexes in the lower extremities, and difficulty with tandem walking.

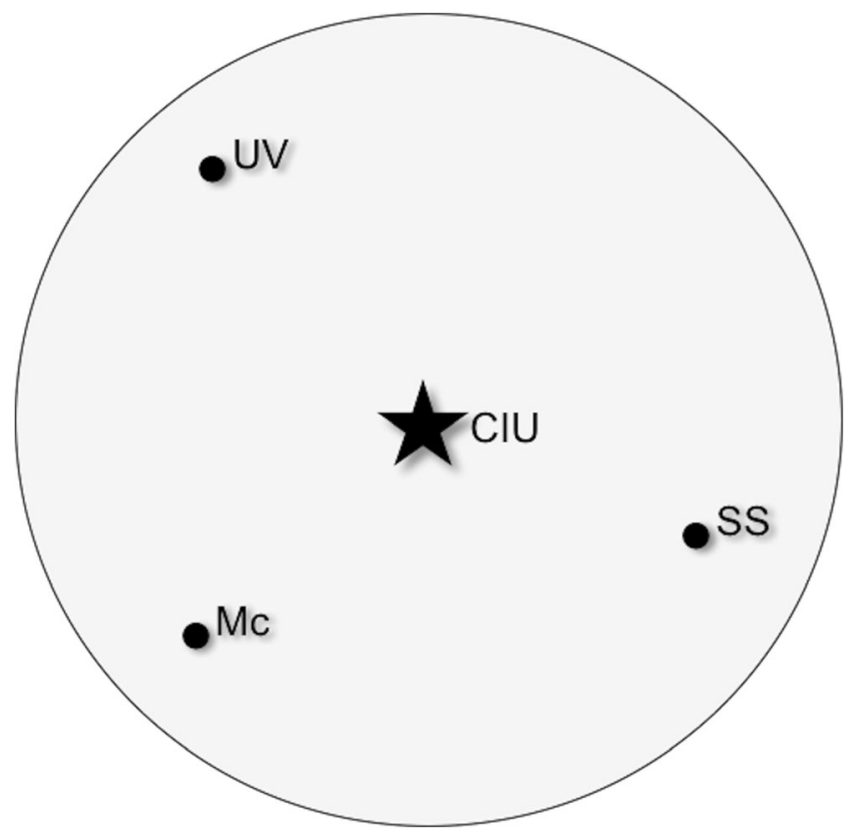

Figure 1 The urticaria cluster: chronic idiopathic urticaria (CIU) is the pivot diagnosis, while rare conditions such as urticarial vasculitis (UV), mastocytosis (Mc), and Schnitzler's syndrome (SS) reside at the edge of the cluster.
A palpable liver edge may be normal with a thin body habitus, but could also reflect downward displacement of the liver or hepatomegaly; the latter arises in the setting of congestion, inflammation, or infiltration of the liver. The absence of jugular venous elevation suggests hepatic congestion is unlikely. The liver is not tender, which makes acute inflammation less likely.

Diabetic polyneuropathy is common and can lead to diminished proprioception and vibration. However, with no other end-organ damage attributed to diabetes (which is well-controlled), diabetic polyneuropathy is unlikely. A paraneoplastic or vasculitic neuropathy is a greater concern. Given impaired vibratory and proprioceptive sensation, posterior spinal column degeneration from neurosyphilis or vitamin B12 deficiency should be considered.

Hepatomegaly, lymphadenopathy, and neuropathy are concerning for an infiltrative disorder. If repeat CT imaging reaffirms the absence of a primary cancer in the chest, abdomen, or pelvis, then lymphoma, breast cancer (which would require mammography), or metastatic cancer of unknown primary would be candidate malignancies. Sarcoidosis would be a suitable explanation, particularly if thoracic lymphadenopathy is prominent on subsequent imaging. IgG4-related disease (IgG4-RD), which frequently involves the nerves, liver, and lymph nodes and causes weight loss, is possible, although fever is atypical.

With this information, the teacher can create a second cluster around hepatomegaly or neuropathy; neuropathy is more likely to explain the previously identified problem of falls, and therefore is chosen as the next cluster to illustrate on the board. The teacher can emphasize to learners that when selecting a pivot, the intent is not to immediately identify the underlying condition, but rather to select a familiar or plausible diagnosis as a starting point for the differential diagnosis. A suitable starting point for this cluster is diabetic neuropathy, which is highly prevalent, although it is less plausible in this case.

The patient had generalized urticarial patches most prominently over her trunk and extremities, sparing the palms and soles. The rash had been intermittently present for the past 5 years. It was mildly pruritic. She was unable to identify triggers, and trials of antihistamines or steroids did not decrease her symptoms.

The mild pruritus and failure to improve with steroids are atypical for allergic urticaria. I would consider systemic disorders that cause fevers, weight loss, hepatomegaly, lymphadenopathy, and neuropathy along with chronic patch or plaque formation (rather than urticaria). Systemic lupus erythematosus and syphilis could explain this constellation and should be tested for. Mycosis fungoides and leprosy can present for many years as non-specific, or errantly characterized, rashes. Given the rash, neuropathy, and sclerotic bone lesions, testing for a monoclonal immunoglobulin is indicated to evaluate the possibility of POEMS [polyneuropathy, 
organomegaly, endocrinopathy, monoclonal gammopathy, and skin changes] syndrome.

A cluster is not an amorphous collection of diagnoses, but is rather a structured representation of the clinician's clinical reasoning and perception of diagnostic probability. The distance of a given diagnosis from the pivot represent its degree of alignment with the patient's clinical presentation. For example, mycosis fungoides and leprosy cause skin changes but are unlikely to be mistaken for urticaria, so the teacher would place them at the edge of the chronic urticaria cluster, with the large distance from the pivot indicating lower likelihood of diagnosis.

As multiple clusters are created, they can be drawn to overlap with one another, highlighting shared diagnoses that unify multiple features of the case. In this way, a teacher can visually encourage students to search for a parsimonious explanation for a collection of findings.

The white cell count was 15,800 per cubic millimeter, with $85 \%$ neutrophils, $12 \%$ lymphocytes, $2 \%$ monocytes, and $1 \%$ eosinophils. The hemoglobin level was $9.7 \mathrm{~g}$ per deciliter, the hematocrit $31 \%$, the mean corpuscular volume 83 femtoliters, and the platelet count 452,000 per cubic milliliter. The peripheral blood smear revealed normocytic, normochromic anemia with neutrophilpredominant leukocytosis. Serum electrolytes, calcium, creatinine, liver tests, and thyroid function tests were normal. Albumin was $3.3 \mathrm{~g} / \mathrm{dL}$. The C-reactive protein (CRP) level was $120 \mathrm{mg}$ per liter (reference range, $<10.9$ ), and the erythrocyte sedimentation rate (ESR) was $87 \mathrm{~mm}$ per hour (reference range, $<\mathbf{2 0}$ ). Bacterial and fungal blood cultures were sterile. Human immunodeficiency virus (HIV) antibody was negative. A tuberculin skin test was non-reactive, and chest radiography showed no cardiopulmonary abnormalities.

Her neutrophilic leukocytosis, normocytic anemia, and elevated CRP and ESR point to chronic inflammation. Although hepatomegaly was detected on exam, there is no elevation of alkaline phosphatase that is characteristic of infiltrative disorders.

There is no diarrhea, hypoalbuminemia, or abdominal pain that would suggest a malabsorptive condition like celiac sprue or Whipple's disease.

Skin biopsy may confirm typical urticarial lesions or reveal urticarial vasculitis, cutaneous lymphoma, or cutaneous lupus. A lymph node biopsy could be examined for tuberculosis, lymphoma, sarcoidosis, IgG4-RD, or mastocytosis. Lymphoma is the leading concern at this point.

Cervical spine MRI revealed mild degenerative changes without spinal canal or foraminal narrowing or cord signal changes. $\mathrm{CT}$ of the chest and abdomen revealed enlarged bilateral axillary lymph nodes (e.g., right axillary node $20 \times 9 \mathrm{~mm}$ ), sub-centimeter lower chest wall lymph nodes, and a liver span of $19 \mathrm{~cm}$. A left axillary lymph node was excised. Pathologic analysis of the specimen showed a reactive lymph node with follicular hyperplasia, minimal expansion of the paracortical regions, and sinus histiocytosis. Flow cytometry of the lymph node showed no monoclonal lymphoid population.

The salient features of this case are as follows: middle age, intermittent fever, profound weight loss, chronic intermittent steroid-resistant rash, hepatomegaly, bilateral axillary lymphadenopathy, neuropathy, neutrophilia, anemia, moderate ANA seropositivity, a sclerotic bone lesion, and elevated markers of inflammation. There is considerable redundancy in this list, with two overarching themes: inflammation and infiltration.

The absence of granulomas excludes sarcoidosis and makes tuberculosis unlikely. The histologic findings of lymphoma and IgG4-RD were not present. Although a cancer has not been identified on imaging or biopsy, malignant plasma cell disorders such as POEMS or AL amyloidosis are possible. However, given the multi-system nature of this illness, an autoimmune disorder or infiltrative disorder is more likely.

The discussant has sufficient information to develop an elaborated problem representation against which specific illness scripts can be compared. Even as the teacher develops specific clusters on the whiteboard, it is important to challenge learners to synthesize the complex clinical data into an overarching problem representation. This step encourages consideration of the larger context in which the clusters are being developed.

The repeat ANA titer was 1:160. The rheumatoid factor level was normal. Levels of anti-cyclic citrullinated peptide, anti-double-stranded DNA, anti-neutrophil cytoplasmic, anti-Ro, anti-La, and anti-Smith antibodies were all within normal limits. $\mathrm{C} 3, \mathrm{C} 4$, and $\mathrm{CH} 50$ levels were normal.

Levels of IgA, IgG, and IgM were normal. Ferritin was $1325 \mathrm{ng} / \mathrm{mL}$ (normal range, 10 to 291). Serum protein electrophoresis demonstrated no paraproteinemia, but serum immunofixation revealed a monoclonal IgM immunoglobulin with kappa specificity. Urine immunofixation detected a monoclonal kappa light chain. Urine protein electrophoresis, serum free light chains, and serum vascular endothelial growth factor were not tested. Nerve conduction studies revealed an axonal sensorimotor polyneuropathy.

Detection of monoclonal proteins by immunofixation but not immunoelectrophoresis suggests a low level of excess kappa-restricted IgM. Monoclonal proteins are produced in excess in a number of plasma cell disorders. Most paraproteinemias are associated with neuropathy; immunoglobulins or amyloid proteins can directly interact with neural antigen targets or form deposits in the nerve.

Multiple myeloma is the most common paraproteinemia and warrants consideration given the anemia; however, the serum creatinine and calcium are normal, and IgM multiple myeloma is exceedingly rare. Waldenstrom's macroglobulinemia is associated with monoclonal IgM; however, that elevation is usually substantial. The gammopathy of POEMS is 
usually lambda chain-restricted, and the neuropathy is usually demyelinating rather than axonal. AL amyloidosis causes neuropathy and organomegaly, but is associated with isolated light-chain excess.

The paraproteinemia resurrects the possibility of Schnitzler's syndrome, which is an autoinflammatory neutrophilic dermatosis characterized by urticaria, fever, bone pain, and IgM gammopathy. Given the chronicity of the rash, the inflammatory nature of this illness, and the IgM monoclonal gammopathy, I favor Schnitzler's syndrome. I am uncertain about the etiology of the neuropathy; it may be paraproteinemia-related.

With the discovery of paraproteinemia, the teacher can open a third cluster on the whiteboard. As with the two previous clusters, the pivot-multiple myeloma-is the diagnosis that comes to mind immediately, either because of its prevalence or its suitability for the case. At this point, the teacher can present the learner with the challenge of identifying a unifying diagnosis that resides in the overlapping space between all three clusters and is compatible with other features of the problem representation (Fig. 2).

Schnitzler's syndrome originated in the urticaria cluster but also resides in the paraproteinemia cluster. Although the cause of neuropathy remains uncertain, the teacher (like the discussant) can analogize from the general concept of paraproteinemic neuropathy to speculate that Schnitzler's syndrome-associated paraproteinemia may be responsible. This allows the teacher to tentatively place Schnitzler's at the edge of the neuropathy cluster until this hypothesis is evaluated.
A bone marrow biopsy revealed normal trilineage hematopoiesis, and flow cytometry showed no evidence of a monoclonal population. The patient was diagnosed with Schnitzler's syndrome by fulfilling the Strasbourg criteria, which include two obligate criteria (IgM monoclonal gammopathy and chronic urticarial rash) and three minor criteria (recurrent fever, bone remodeling, and leukocytosis).

Following daily subcutaneous injections of the IL-1 antagonist anakinra, the patient's fatigue, fever, and rash quickly improved. After 1 week of therapy, the CRP and white blood cell count were $1.3 \mathrm{mg}$ per liter and 6400 per cubic millimeter, respectively. After six months of therapy, her weight had increased from 124 to 164 pounds. After 1 year of daily therapy, she had no symptoms or laboratory findings of Schnitzler's syndrome except for persistent IgM monoclonal gammopathy. Her gait instability resolved, and she has had no additional falls.

\section{DISCUSSION}

There is no evidence-based method for teachers to visually represent a clinician's approach to reaching a diagnosis in a complex case. Common techniques that teachers use at the whiteboard include listing differential diagnoses for key problems or emphasizing their intersection using Venn diagrams. This case illustrates how educators can use the pivot and cluster strategy (PCS) to add more detail in visually representing and teaching the diagnostic process. As described by Shimizu and Tokuda, PCS captures the

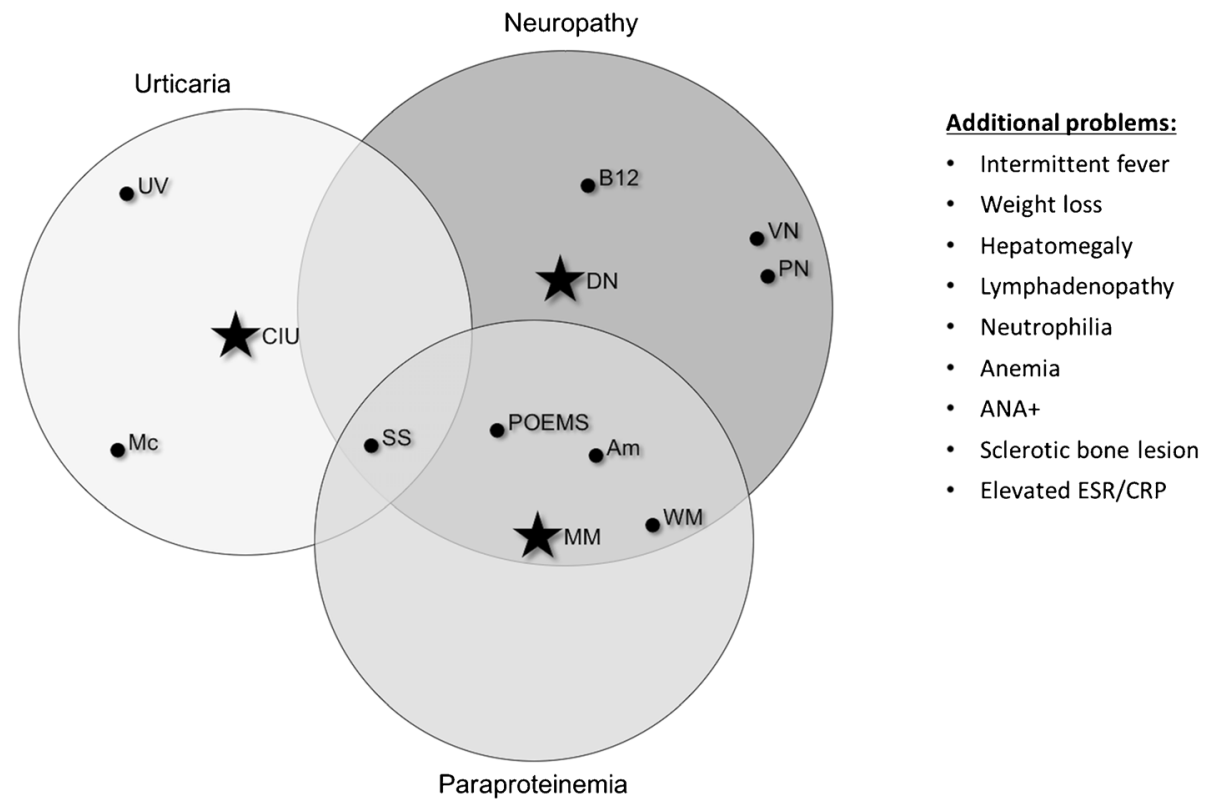

Figure 2 The interplay between diagnostic clusters guides the diagnostic process. The pivot of the neuropathy cluster is diabetic neuropathy (DN), which is surrounded by vitamin B12 deficiency (B12), paraneoplastic neuropathy (PN), and vasculitic neuropathy (VN). The pivot of the paraproteinemia cluster is multiple myeloma (MM); three cluster components—amyloidosis (Am), Waldenstrom's macroglobulinemia (WM), and POEMS - also fit within the neuropathy cluster. Schnitzler's syndrome is the only diagnosis that resides in the overlap between all three clusters and explains the other salient features of the case, listed on the right. 
clinician's approach of identifying a common or plausible diagnosis, designated the "pivot," and formulating a "cluster" of related diagnoses around it. ${ }^{1}$

When considering a complex case, identification of a pivot diagnosis for one finding is a more accessible starting point for a learner than immediately listing a comprehensive differential diagnosis. After assessing the fit of the pivot diagnosis (often something common like diabetic neuropathy or multiple myeloma), the learner is challenged to build a cluster of alternative diagnoses which is diagramed on the whiteboard.

The teacher can help the learner assign a hypothetical distance from the pivot of each alternative diagnosis to represent its degree of alignment with the patient's clinical presentation. In this way, PCS transforms a one-dimensional list of differential diagnoses into a two-dimensional visual representation of the plausible diagnoses and their relative likelihoods. Figure 1 represents the discussant's thoughts through the architecture of a diagnostic cluster. Schnitzler's syndrome, urticarial vasculitis, and mastocytosis are three rare causes of urticaria that are placed at a substantial distance from the pivot of chronic idiopathic urticaria.

In this case, while tracking the background issue of undifferentiated inflammation, two additional clusters were identified: neuropathy and paraproteinemia. The discussant recognized that Schnitzler's syndrome sat at the edge of the urticaria and paraproteinemia clusters. Although he could not confidently place Schnitzler's syndrome in the neuropathy cluster, he drew upon the concept of paraproteinemic neuropathy ${ }^{2}$ to propose Schnitzler's syndrome as a plausible unifying diagnosis. The resolution of the patient's neuropathy following anakinra treatment triggered further literature review, which revealed that neuropathy was seen in $7 \%$ of Schnitzler's syndrome cases in one series and may be part of the disease spectrum. ${ }^{3,4}$

By building one cluster at a time and considering the overlap between them, the teacher can guide learners to a rare diagnosis while simultaneously illustrating diagnostic reasoning concepts that can be employed in any clinical scenario. This approach empowers learners to develop their own clinical reasoning skills and understand that by returning to the drawing board, they can tackle any diagnostic challenge.

\section{CLINICAL TEACHING POINTS}

1. Recurrent wheals (hives) are usually a manifestation of chronic urticaria which is usually idiopathic but sometimes can be attributed to drug, food, or other environmental exposures.

2. Unlike histamine-mediated urticaria, autoinflammatorymediated urticaria tends to be minimally pruritic and symmetrically distributed, and does not improve with steroids. $^{5}$

3. Autoinflammatory disorders (e.g., Schnitzler's syndrome, adult-onset Still's disease, cryopyrin-associated periodic syndrome) are rare diseases that arise from antigen-independent activation of the innate immune system, in contrast to autoimmune diseases which arise from antigen-dependent activation of the adaptive immune system.

4. Schnitzler's syndrome is characterized by urticaria, a monoclonal IgM gammopathy, fever, sclerotic bone lesions, leukocytosis, and elevated CRP. A minority of cases feature neuropathy.

5. Treatment with anakinra, an interleukin-1 receptor antagonist, suppresses the overactive innate immune system and achieves complete resolution of symptoms in up to $96 \%$ of Schnitzler's syndrome cases. ${ }^{6}$

Acknowledgements: This case was presented at the annual meeting of the 2016 Alabama chapter of the American College of Physicians (ACP) and at the 2017 national ACP meeting.

Corresponding Author: Jori E. May, MD; Department of Medicine University of Alabama at Birmingham, Birmingham, AL, USA (e-mail: jemay@uabmc.edu).

\section{Compliance with Ethical Standards:}

Conflict of Interest: Drs. May, Blackburn, and Centor declare that they have no conflict of interest. Dr. Dhaliwal reports receiving honoraria from ISMIE Mutual Insurance Company and Physicians' Reciprocal Insurers.

\section{REFERENCES}

1. Shimizu T, Tokuda Y. Pivot and cluster strategy: a preventive measure against diagnostic errors. Int J Gen Med. 2012;5:917-21.

2. Rajabally YA. Neuropathy and paraproteins: review of a complex association. Eur J Neurol. 2011;18(11):1291-8.

3. De Koning HD. Schnitzler's syndrome: lessons from 281 cases. Clin Transl Allergy. 2014;4:41.

4. Lebbe C, Rybojad M, Klein F, et al. Schnitzler's syndrome associated with sensorimotor neuropathy. J Am Acad Dermatol. 1994;30(2 Pt 2):316-8.

5. Krause $\mathbf{K}$, Grattan $\mathbf{C E}$, Bindslev-Jensen $\mathbf{C}$, et al. How not to miss autoinflammatory diseases masquerading as urticaria. Allergy. 2012;67(12): 1465-74.

6. Simon A, Asli B, Braun-Falco M, et al. Schnitzler's syndrome: diagnosis, treatment, and follow-up. Allergy. 2013;68(5):562-8. 